\title{
A Preconditioner for the Schur Complement Matrix
}

\author{
M. Storti*, \\ L. Dalcín,R. Paz,A. Yommi,V. Sonzogni and N. Nigro \\ Centro Internacional de Métodos Computacionales en Ingeniería (CIMEC), \\ INTEC(CONICET-UNL), Güemes 3450, (3000) Santa Fe, Argentina.
}

\begin{abstract}
A preconditioner for iterative solution of the interface problem in Schur Complement Domain Decomposition Methods is presented. This preconditioner is based on solving a global problem in a narrow strip around the interface. It requires much less memory and computing time than classical Neumann-Neumann preconditioner and its variants, and handles correctly the flux splitting among subdomains that share the interface. The aim of this work is to present a theoretical basis (regarding the behavior of Schur complement matrix spectra) and some simple numerical experiments conducted in a sequential environment as a motivation for adopting the proposed preconditioner. Efficiency, scalability, and implementation details on a production parallel finite element code $[1,2]$ can be found in works $[3,4]$.
\end{abstract}

Key words: domain decomposition methods, Schur complement preconditioning

\section{INTRODUCTION}

Linear systems obtained from discretization of PDE's by means of Finite Difference or Finite Element Methods are normally solved in parallel by iterative methods [5] because they are much less coupled than direct solvers.

The Schur complement domain decomposition method leads to a reduced system better suited for iterative solution than the global system, since its condition number is lower $\left(\propto 1 / h\right.$ vs. $\propto 1 / h^{2}$ for the global system, $h$ being the

* Corresponding author.

Email address: mstorti@intec.unl.edu.ar (M. Storti).

URL: http://www.cimec.org.ar/mstorti (M. Storti). 
mesh size) and the computational cost per iteration is not so high once the subdomain matrices have been factorized. In addition, it has other advantages over global iteration. It solves bad "inter-equation" conditioning, it can handle Lagrange multipliers and in a sense it can be thought as a mixture between a global direct solver and a global iterative one.

The efficiency of iterative methods can be further improved by using preconditioners [6]. For mechanical problems, Neumann-Neumann is the most classical one. From a mathematical point of view, the preconditioner is defined by approximating the inverse of the global Schur complement matrix by the weighted sum of local Schur complement matrices. From a physical point of view, Neumann-Neumann preconditioner is based on splitting the flux applied to the interface in the preconditioning step and solving local Neumann problems in each subdomain. This strategy is good only for symmetric operators.

We propose a preconditioner based on solving a global problem in a "strip" of nodes around the interface. A similar idea has been already exploited in the context of FETI methods [7] in order to construct an approximation of local Schur complement matrices. In contrast, the preconditioning technique considered here approximates the inverse of global Schur matrix. This preconditioner performs better for non-symmetric operators, it does not suffer from the rigid body modes for internal floating subdomains as is the case for the Neumann-Neumann preconditioner and naturally conducts to subdomain coupling (thus eliminating the need of a coarse problem). When the width of the strip is narrow, the computational cost and memory requirements are low and the iteration count is relatively high, when the strip is wide, the converse is verified. A detailed computation of the eigenvalue spectra for simple cases and some numerical examples are presented.

\section{SCHUR COMPLEMENT DOMAIN DECOMPOSITION METHOD}

It is clear that knowing the eigenvalue spectrum of the Schur complement matrix is one of the most important issues in order to develop suitable preconditioners. To obtain analytical expressions for Schur complement matrix eigenvalues and also the influence of several preconditioners, we consider a simplified problem, namely the solution to the Poisson problem in a unit square:

$$
\Delta \phi=g, \quad \text { in } \Omega=\{0<x, y<1\}
$$

with boundary conditions

$$
\phi=\bar{\phi}, \quad \text { at } \Gamma=\{x, y=0,1\} .
$$


where $\phi$ is the unknown, $g(x, y)$ is a given source term and $\Gamma$ is the boundary. Consider now the partition of $\Omega$ in $N_{\mathrm{s}}$ non-overlapping subdomains $\Omega_{1}, \Omega_{2}$, $\ldots, \Omega_{N_{\mathrm{s}}}$, such that $\Omega=\Omega_{1} \cup \Omega_{2} \cup \ldots \cup \Omega_{N_{\mathrm{s}}}$. For the sake of simplicity, we assume that the subdomains are rectangles of unit height and width $L_{j}$. In practice this is not the best partition, but it will allow us to compute the eigenvalues of the interface problem in closed form. Let $\Gamma_{\mathrm{int}}=\Gamma_{1} \cup \Gamma_{2} \cup \ldots \cup \Gamma_{N_{\mathrm{s}}-1}$ be the interior interfaces among adjacent subdomains. Given a guess $\psi_{j}$ for the trace of $\phi$ in the interior subdomains $\left.\phi\right|_{\Gamma_{j}}$, we can solve each interior problem independently as

$$
\begin{aligned}
\Delta \phi & =g, \quad \text { in } \Omega_{j}, \\
\phi & = \begin{cases}\psi_{j-1}, & \text { at } \Gamma_{j-1}, \\
\psi_{j}, & \text { at } \Gamma_{j}, \\
\bar{\phi}, & \text { at } \Gamma_{\mathrm{up}, j}+\Gamma_{\text {down }, j},\end{cases}
\end{aligned}
$$

where $\psi_{0}=\left.\bar{\phi}\right|_{x=0}$ and $\psi_{N_{\mathrm{s}}}=\left.\bar{\phi}\right|_{x=1}$ are given.

\subsection{The Steklov operator}

Not all combinations of trace values $\left\{\psi_{j}\right\}$ give the solution of the original problem (1). Indeed, the solution to (1) is obtained when the trace values are chosen in such a way that the flux balance condition at the internal interfaces is satisfied,

$$
f_{j}=\left.\frac{\partial \phi}{\partial x}\right|_{\Gamma_{j}} ^{-}-\left.\frac{\partial \phi}{\partial x}\right|_{\Gamma_{j}} ^{+}=0,
$$

where the \pm superscripts stand for the derivative taken from the left and right sides of the interface. We can think of the correspondence between the ensemble of interface values $\boldsymbol{\psi}=\left\{\psi_{1}, \ldots, \psi_{N_{\mathrm{s}}-1}\right\}$ and the ensemble of flux imbalances $\mathbf{f}=\left\{f_{1}, \ldots, f_{N_{\mathrm{s}}-1}\right\}$ as an interface operator $\mathcal{S}$ such that

$$
\mathcal{S} \psi=\mathbf{f}-\mathbf{f}_{0},
$$

where all inhomogeneities coming from the source term and Dirichlet boundary conditions are concentrated in the constant term $\mathbf{f}_{0}$, and the homogeneous operator $\mathcal{S}$ is equivalent to solving the equation set (3) with source term $g=0$ and homogeneous Dirichlet boundary conditions $\bar{\phi}=0$ at the external boundary $\Gamma$.

Here, $\mathcal{S}$ is the Steklov operator. In a more general setting, it relates the unknown values and fluxes at boundaries when the internal domain is in equilibrium. In the case of internal boundaries, it can be generalized by replacing the fluxes by the flux imbalances. The Schur complement matrix is a discrete version of the Steklov operator, and we will show that in this simplified case 
we can compute the Steklov operator eigenvalues in closed form, and then a good estimate for the corresponding Schur complement matrix ones.

\subsection{Eigenvalues of Steklov operator}

We will further assume that only two subdomains are present, one of them at the left of width $L_{1}$ and the other at the right of width $L_{2}$, so that $L=$ $L_{1}+L_{2}=1$ is the side length.

We solve first the Laplace problem in each subdomain with homogeneous Dirichlet boundary condition at the external boundary and $\psi$ at the interface,

$$
\begin{aligned}
& \Delta \phi=0, \quad \text { in } \Omega_{1,2}, \\
& \phi= \begin{cases}0, & \text { at } \Gamma, \\
\psi, & \text { at } \Gamma_{1} .\end{cases}
\end{aligned}
$$

The solution of (6) can be expressed as a linear combination of functions of the form

$$
\phi_{n}(x, y)= \begin{cases}{\left[\sinh \left(k_{n} x\right) / \sinh \left(k_{n} L_{1}\right)\right] \sin \left(k_{n} y\right),} & 0 \leq x \leq L_{1}, \\ {\left[\sinh \left(k_{n}(L-x)\right) / \sinh \left(k_{n} L_{2}\right)\right] \sin \left(k_{n} y\right),} & L_{1} \leq x \leq L,\end{cases}
$$

where the wave number $k_{n}$ and the wavelength $\lambda_{n}$ are defined as

$$
k_{n}=2 \pi / \lambda_{n}, \quad \lambda_{n}=2 L / n, \quad n=1, \ldots, \infty .
$$

The flux imbalance for each function in (7) can be computed as

$$
\begin{aligned}
f_{n} & =\left.\frac{\partial \phi_{n}}{\partial x}\right|_{x=L_{1}^{-}}-\left.\frac{\partial \phi_{n}}{\partial x}\right|_{x=L_{1}^{+}}= \\
& =k_{n}\left(\frac{\cosh \left(k_{n} L_{1}\right)}{\sinh \left(k_{n} L_{1}\right)}+\frac{\cosh \left(k_{n} L_{2}\right)}{\sinh \left(k_{n} L_{2}\right)}\right) \sin \left(k_{n} y\right)= \\
& =k_{n}\left[\operatorname{coth}\left(k_{n} L_{1}\right)+\operatorname{coth}\left(k_{n} L_{2}\right)\right] \sin \left(k_{n} y\right) .
\end{aligned}
$$

A given interface value function $\psi$ is an eigenfunction of the Steklov operator if the corresponding flux imbalance $f=\mathcal{S} \psi$ is proportional to $\psi$, i.e. $\mathcal{S} \psi=\omega \psi$, $\omega$ being the corresponding eigenvalue. We can see from (6) to (9) that the eigenfunctions of the Steklov operator are

$$
\psi_{n}(y)=\sin \left(k_{n} y\right)
$$


with eigenvalues

$$
\begin{aligned}
\omega_{n} & =\operatorname{eig}(\mathcal{S})_{n}=\operatorname{eig}\left(\mathcal{S}^{-}\right)_{n}+\operatorname{eig}\left(\mathcal{S}^{+}\right)_{n}= \\
& =k_{n}\left[\operatorname{coth}\left(k_{n} L_{1}\right)+\operatorname{coth}\left(k_{n} L_{2}\right)\right],
\end{aligned}
$$

where $\mathcal{S}^{\mp}$ are the Steklov operators of the left and right subdomains,

$$
\mathcal{S}^{\mp} \psi= \pm\left.\frac{\partial \phi}{\partial x}\right|_{L_{1}^{\mp}},
$$

and their eigenvalues are

$$
\operatorname{eig}\left(\mathcal{S}^{\mp}\right)_{n}=k_{n} \operatorname{coth}\left(k_{n} L_{1,2}\right)
$$

For large $n$, the hyperbolic cotangents in (13) both tend to unity. This shows that the eigenvalues of the Steklov operator grow proportionally to $n$ for large $n$, and then its condition number is infinity. However, when considering the discrete case the wave number $k_{n}$ is limited by the largest frequency that can be represented by the mesh, which is $k_{\max }=\pi / h$ where $h$ is the mesh spacing. The maximum eigenvalue is

$$
\omega_{\max }=2 k_{\max }=\frac{2 \pi}{h}
$$

which grows proportionally to $1 / h$. As the lowest eigenvalue is independent of $h$, this means that the condition number of the Schur complement matrix grows as $1 / h$. Note that the condition number of the discrete Laplace operator typically grows as $1 / h^{2}$. Of course, this reduction in the condition number is not directly translated to total computation time, since we have to take account of the factorization of the subdomain matrices and forward and backward substitutions involved in each iteration to solve internal problems. However, the overall balance is positive and reduction in the condition number, besides being inherently parallel, turns out to be one of the main strengths of domain decomposition methods.

In figure 1 we can see the first and tenth eigenfunctions computed directly from the Schur complement matrix for a 2 subdomain partition, whereas in figure 2 we see the first and twenty-fourth eigenfunction for a 9 subdomain partition. The eigenvalue magnitude is related to eigenfunction frequency along the inter-subdomain interface, and the penetration of the eigenfunctions towards subdomains interiors decays strongly for higher modes. 


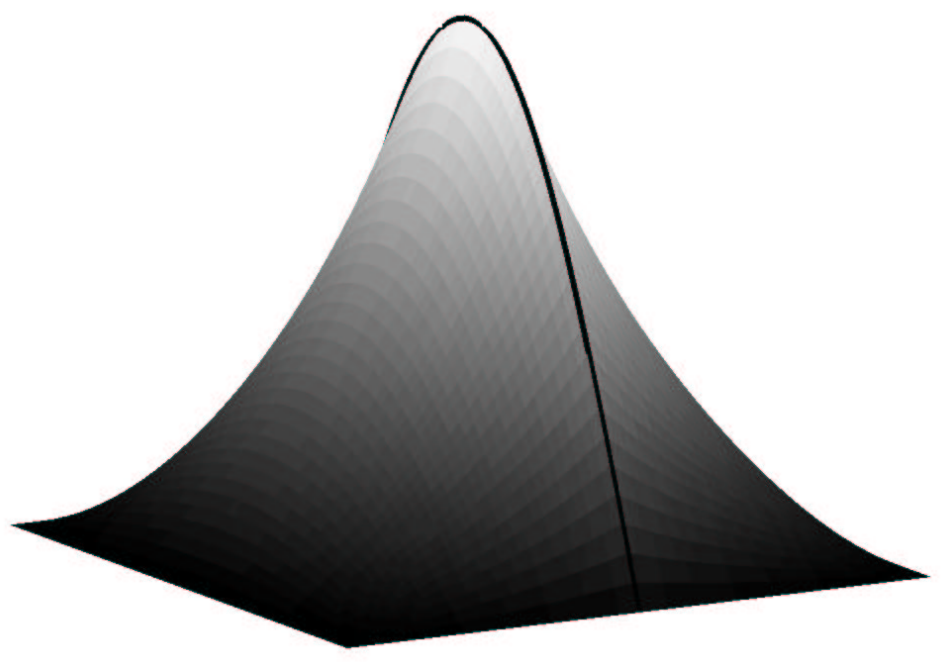

(a) 1-st eigenfunction

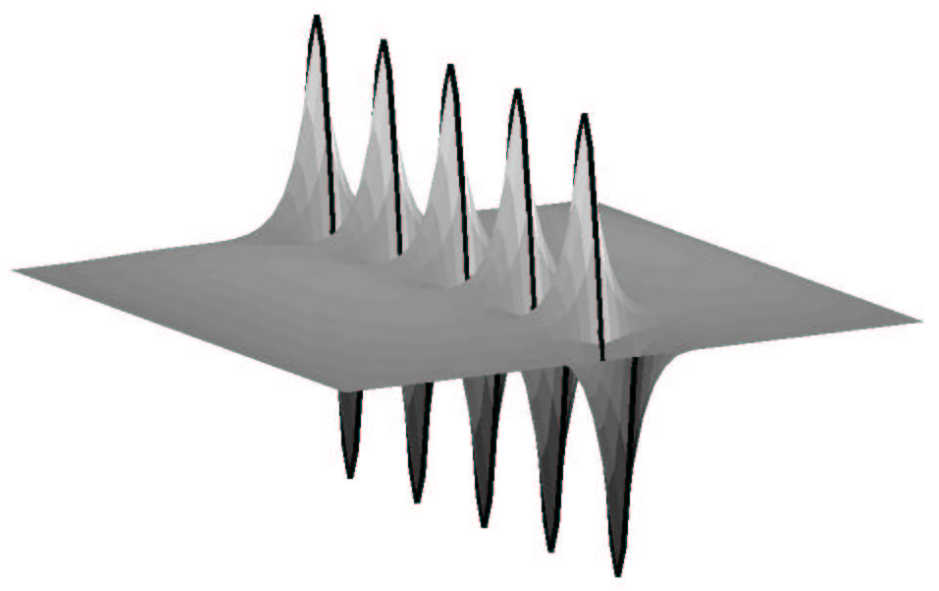

(b) 10-th eigenfunction

Fig. 1. Eigenfunctions of Schur complement matrix with 2 subdomains.

\section{PRECONDITIONERS FOR THE SCHUR COMPLEMENT MA- TRIX}

In order to further improve the efficiency of iterative methods, a preconditioner has to be added so that the condition number of the Schur complement matrix is lowered. The most known preconditioners for mechanical problems are Neumann-Neumann and its variants [8-10] for Schur complements methods, and Dirichlet for FETI methods and its variants [11-14,7]. It can be proved that they reduce the condition number of the preconditioned operator to $O(1)$ 


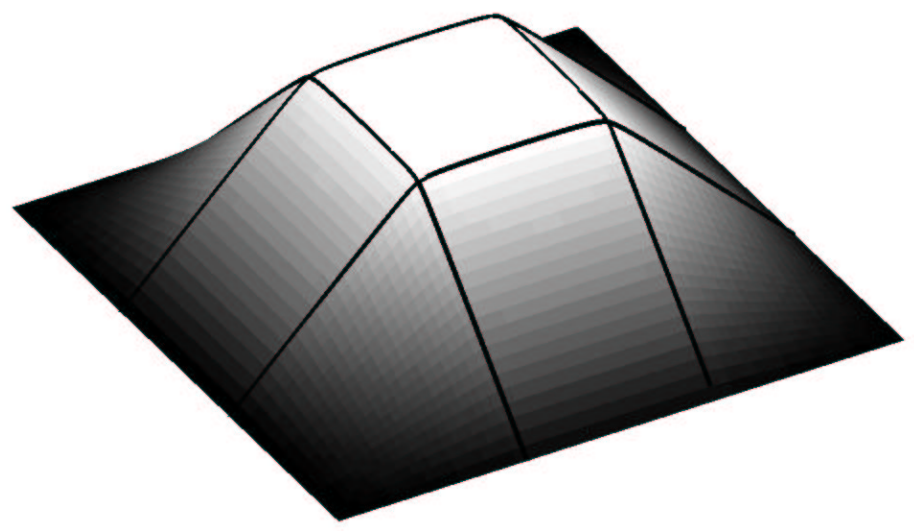

(a) 1-st eigenfunction

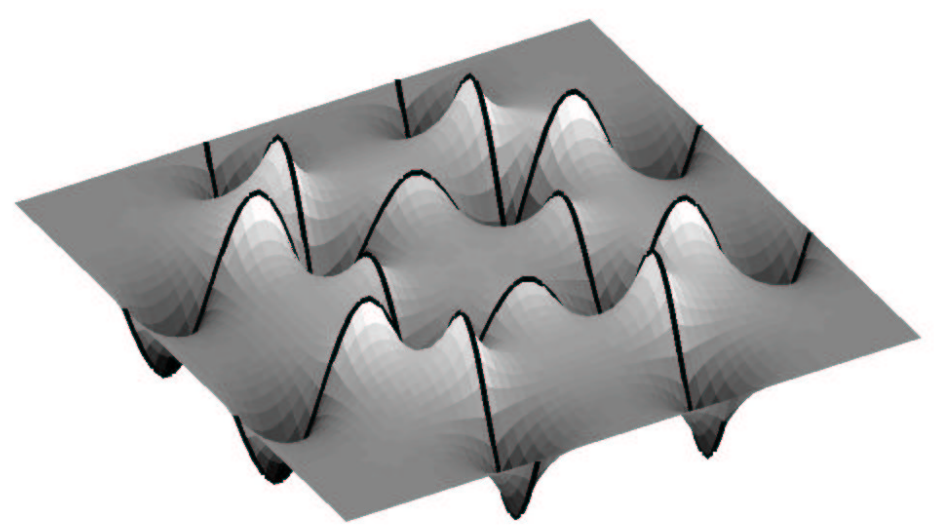

(b) 10-th eigenfunction

Fig. 2. Eigenfunctions of Schur complement matrix with 9 subdomains.

(i.e. independent of $h$ ) in some special cases.

\subsection{The Neumann-Neumann preconditioner}

Consider the Neumann-Neumann preconditioner

$$
\mathcal{P}_{\mathrm{NN}} v=f,
$$

where

$$
v(y)=1 / 2\left[v_{1}\left(L_{1}, y\right)+v_{2}\left(L_{1}, y\right)\right],
$$


and $v_{i}, i=1,2$, are defined through the following problems

$$
\begin{aligned}
\Delta v_{i} & =0 & & \text { in } \Omega_{i}, \\
v_{i} & =0 & & \text { at } \Gamma_{0}+\Gamma_{\mathrm{up}, i}+\Gamma_{\text {down }, i}, \\
(-1)^{i-1} \frac{\partial v_{i}}{\partial x} & =1 / 2 f & & \text { at } \Gamma_{1} .
\end{aligned}
$$

The preconditioner consists in assuming that the flux imbalance $f$ is applied on the interface. Since the operator is symmetric and the domain properties are homogeneous, this "load" is equally split among the two subdomains. Then, we have a problem in each subdomain with the same boundary conditions in the exterior boundaries, and a non-homogeneous Neumann boundary condition at the inter-subdomain interface.

Again, we will show that the eigenfunctions of the Neumann-Neumann preconditioner are (10). Effectively, we can propose for $v_{1}$ the form

$$
v_{1}=C \sinh \left(k_{n} x\right) \sin \left(k_{n} y\right),
$$

where $C$ is determined from the boundary condition at the interface in (17) and results in

$$
C=\frac{1}{2 k_{n} \cosh \left(k_{n} L_{1}\right)},
$$

and similarly for $v_{2}$, so that

$$
\begin{aligned}
& v_{1}(x, y)=\frac{1}{2 k_{n}} \frac{\sinh \left(k_{n} x\right)}{\cosh \left(k_{n} L_{1}\right)} \sin \left(k_{n} y\right) \\
& v_{2}(x, y)=\frac{1}{2 k_{n}} \frac{\sinh \left(k_{n}(L-x)\right)}{\cosh \left(k_{n} L_{2}\right)} \sin \left(k_{n} y\right)
\end{aligned}
$$

Then, the value of $v=\mathcal{P}_{\mathrm{NN}}^{-1} f$ can be obtained from (16)

$$
v(y)=\mathcal{P}_{\mathrm{NN}}^{-1} f=\frac{1}{4 k_{n}}\left[\tanh \left(k_{n} L_{1}\right)+\tanh \left(k_{n} L_{2}\right)\right] \sin \left(k_{n} y\right),
$$

so that the eigenvalues of $\mathcal{P}_{\mathrm{NN}}$ are

$$
\operatorname{eig}\left(\mathcal{P}_{\mathrm{NN}}\right)_{n}=4 k_{n}\left[\tanh \left(k_{n} L_{1}\right)+\tanh \left(k_{n} L_{2}\right)\right]^{-1} .
$$

As its definition suggests, it can be verified that

$$
\operatorname{eig}\left(\mathcal{P}_{\mathrm{NN}}\right)_{n}=4\left[\operatorname{eig}\left(\mathcal{S}^{-}\right)_{n}^{-1}+\operatorname{eig}\left(\mathcal{S}^{+}\right)_{n}^{-1}\right]^{-1}
$$

As the Neumann-Neumann preconditioner (15) and the Steklov operator (5) diagonalize in the same basis (10) (i.e., they "commute"), the eigenvalues of 
the preconditioned operator are simply the quotients of the respective eigenvalues, i.e.

$$
\operatorname{eig}\left(\mathcal{P}_{\mathrm{NN}}^{-1} \mathcal{S}\right)_{n}=1 / 4\left[\tanh \left(k_{n} L_{1}\right)+\tanh \left(k_{n} L_{2}\right)\right]\left[\operatorname{coth}\left(k_{n} L_{1}\right)+\operatorname{coth}\left(k_{n} L_{2}\right)\right] .
$$

We see that all $\tanh \left(k_{n} L_{j}\right)$ and $\operatorname{coth}\left(k_{n} L_{j}\right)$ factors tend to unity for $n \rightarrow \infty$, then we have

$$
\operatorname{eig}\left(\mathcal{P}_{\mathrm{NN}}^{-1} \mathcal{S}\right)_{n} \rightarrow 1 \quad \text { for } n \rightarrow \infty
$$

so that this means that the preconditioned operator $\mathcal{P}_{\mathrm{NN}}^{-1} \mathcal{S}$ has a condition number $O(1)$, i.e. it doesn't degrade with mesh refinement. This is optimal, and is a well known feature of the Neumann-Neumann preconditioner. In fact, for a symmetric decomposition of the domain (i.e. $L_{1}=L_{2}=1 / 2$ ), we have

$$
\operatorname{eig}\left(\mathcal{P}_{\mathrm{NN}}^{-1} \mathcal{S}\right)_{n}=\frac{1}{4} 2 \tanh \left(k_{n} / 2\right) 2 \operatorname{coth}\left(k_{n} / 2\right)=1,
$$

so that the preconditioner is equal to the operator and convergence is achieved in one iteration.

Note that comparing (11) and (23) we can see that the preconditioning is good as long as

$$
\operatorname{eig}\left(\mathcal{S}^{-}\right)_{n} \approx \operatorname{eig}\left(\mathcal{S}^{+}\right)_{n}
$$

This is true for symmetric operators and symmetric domain partitions (i.e. $\left.L_{1} \approx L_{2}\right)$. Even for $L_{1} \neq L_{2}$, if the operator is symmetric, then (27) is valid for large eigenvalues. However, this fails for non-symmetric operators as in the advection-diffusion case, and also for irregular interfaces.

Another aspect of the Neumann-Neumann preconditioner is the occurrence of indefinite internal Neumann problems, which leads to the need of solving a coarse problem $[8,9]$ in order to solve the "rigid body modes" for internal floating subdomains. The coarse problem couples the subdomains and hence ensures scalability when the number of subdomains increases. However, this adds to the computational cost of the preconditioner.

\subsection{The Interface Strip (IS) preconditioner}

A key point about the Steklov operator is that its high frequency eigenfunctions decay very strongly far from the interface, so that a preconditioning that represents correctly the high frequency modes can be constructed if we solve a problem on a narrow strip around the interface. In fact, the $n$-th eigenfunction with wave number $k_{n}$ given by (7) decays far from the interface as $\exp \left(-k_{n}|s|\right)$ where $s$ is the distance to the interface. Then, this high frequency modes will be correctly represented if we solve a problem on a strip of width $b$ around the interface, provided that the interface width is large with respect to the mode wave length $\lambda_{n}$. 
The "Interface Strip Preconditioner" (ISP) is defined as

$$
\mathcal{P}_{\text {IS }} v=f
$$

where

$$
f=\left.\frac{\partial w}{\partial x}\right|_{x=L_{1}^{-}}-\left.\frac{\partial w}{\partial x}\right|_{x=L_{1}^{+}}
$$

and

$$
\begin{aligned}
\Delta w=0 & \text { in } 0<\left|x-L_{1}\right|<b \text { and } 0 \leq x \leq 1, \\
w=0 & \text { at }\left|x-L_{1}\right|=b \text { or } y=0,1, \\
w=v & \text { at } x=L_{1} .
\end{aligned}
$$

Please note that for high frequencies (i.e. $k_{n} b$ large) the eigenfunctions of the Steklov operator are negligible at the border of the strip, so that the boundary condition at $\left|x-L_{1}\right|=b$ is justified. The eigenfunctions for this preconditioner are again given by (10) and the eigenvalues can be taken from (11), replacing $L_{1,2}$ by $b$, i.e.

$$
\operatorname{eig}\left(\mathcal{P}_{\mathrm{IS}}\right)_{n}=2 \operatorname{eig}\left(\mathcal{S}_{b}\right)_{n}=2 k_{n} \operatorname{coth}\left(k_{n} b\right),
$$

where $\mathcal{S}_{b}$ is the Steklov operator corresponding to a strip of width $b$.

For the preconditioned Steklov operator, we have

$$
\operatorname{eig}\left(\mathcal{P}_{\mathrm{IS}}^{-1} \mathcal{S}\right)_{n}=1 / 2 \tanh \left(k_{n} b\right)\left[\operatorname{coth}\left(k_{n} L_{1}\right)+\operatorname{coth}\left(k_{n} L_{2}\right)\right]
$$

We note that $\operatorname{eig}\left(\mathcal{P}_{\mathrm{IS}}^{-1} \mathcal{S}\right)_{n} \rightarrow 1$ for $n \rightarrow \infty$, so that the preconditioner is optimal, independently of $b$. Also, for $b$ large enough we recover the original problem so that the preconditioner is exact (convergence is achieved in one iteration). However, in this case the use of this preconditioner is impractical, since it implies solving the whole problem. Note that in order to solve the problem for $v$, we need information from both sides of the interface, while the Neumann-Neumann preconditioner can be solved independently in each subdomain. This is a disadvantage in terms of efficiency, since we have to waste communication time in sending the matrix coefficients in the strip from one side to the other or otherwise compute them in both processors. However, we will see that efficient preconditioning can be achieved with few node layers and negligible communication. Moreover, we can solve the preconditioner problem by iteration, so that no migration of coefficients is needed.

\section{THE ADVECTIVE-DIFFUSIVE CASE}

Consider now the advective diffusive case,

$$
\kappa \Delta \phi-u \phi_{, x}=g \text { in } \Omega
$$


where $\kappa$ is the thermal conductivity of the medium and $u$ the advection velocity. The problem can be treated in a similar way, and the Steklov operators are defined as

where

$$
\mathcal{S}^{\mp} \psi= \pm\left.\phi_{, x}\right|_{L_{1}^{\mp}}
$$

$$
\begin{aligned}
\kappa \Delta \phi-u \phi_{, x} & =0 \quad \text { in } \Omega_{1,2}, \\
\phi & = \begin{cases}0 & \text { at } \Gamma, \\
\psi & \text { at } \Gamma_{1} .\end{cases}
\end{aligned}
$$

The eigenfunctions are still given by (10). Looking for solutions of the form $v \propto \exp (\mu x) \sin \left(k_{n} y\right)$ we obtain a constant coefficient second order differential equation with characteristic polynomial

$$
\kappa \mu^{2}-u \mu-\kappa k_{n}^{2}=0
$$

whose roots are

$$
\mu^{ \pm}=\frac{u \pm \sqrt{u^{2}+4 \kappa^{2} k_{n}^{2}}}{2 \kappa}=\frac{u}{2 \kappa} \pm \delta_{n}
$$

After some algebra, the solution of (35) is

$$
\phi_{n}= \begin{cases}\mathrm{e}^{u\left(x-L_{1}\right) / 2 \kappa} \frac{\sinh \left(\delta_{n} x\right)}{\sinh \left(\delta L_{1}\right)} \sin \left(k_{n} y\right) & \text { for } 0 \leq x \leq L_{1}, 0 \leq y \leq L, \\ \mathrm{e}^{u\left(x-L_{1}\right) / 2 \kappa \frac{\sinh \left(\delta_{n}(L-x)\right)}{\sinh \left(\delta L_{2}\right)} \sin \left(k_{n} y\right)} & \text { for } L_{1} \leq x \leq L, 0 \leq y \leq L,\end{cases}
$$

and the eigenvalues are then

$$
\begin{aligned}
\operatorname{eig}\left(\mathcal{S}^{-}\right)_{n} & =\frac{u}{2 \kappa}+\delta_{n} \operatorname{coth}\left(\delta_{n} L_{1}\right) \\
\operatorname{eig}\left(\mathcal{S}^{+}\right)_{n} & =-\frac{u}{2 \kappa}+\delta_{n} \operatorname{coth}\left(\delta_{n} L_{2}\right)
\end{aligned}
$$

In figure 3 we see the first and tenth eigenfunctions for a problem with an advection term at a global Pèclet number of $\mathrm{Pe}=u L / 2 \kappa=2.5$. For low frequency modes, advective effects are more pronounced and the first eigenfunction (on the left) is notably biased to the right. In contrast, for high frequency modes (like the tenth mode shown at the right) the diffusive term prevails and the eigenfunction is more symmetric about the interface, and (as in the pure diffusive case) concentrated around it. Note that now the eigenvalues for the right and left part of the Steklov operator may be very different due to the asymmetry introduced by the advective term. This difference in splitting is more important for the lowest mode.

In figures 4 to 7 we see the eigenvalues as a function of the wave number $k_{n}$. Note that for a given side length $L$ only a certain sequence of wave numbers, given by (8) should be considered. However, it is perhaps easier to consider the continuous dependence of the different eigenvalues upon the wave number $k$. 


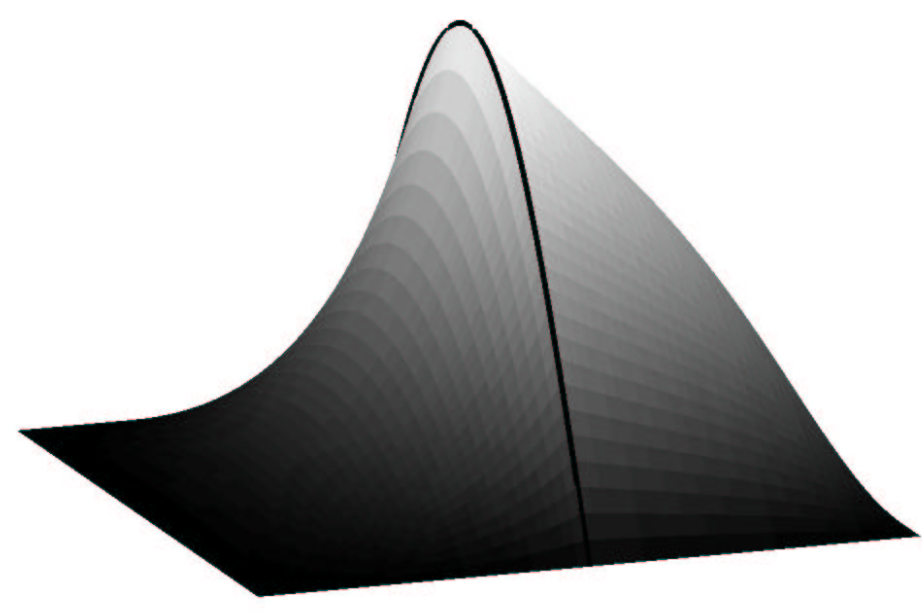

(a) 1-st eigenfunction

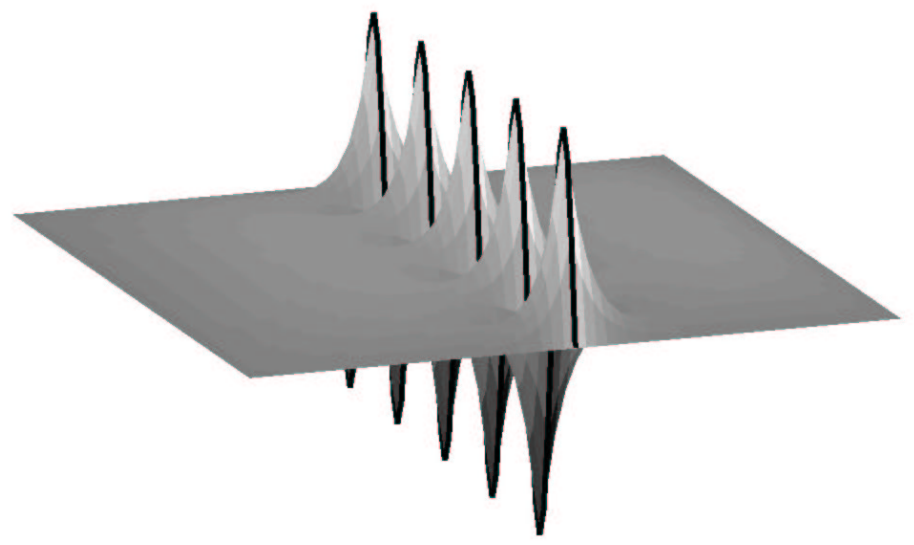

(b) 10-th eigenfunction

Fig. 3. Eigenfunctions of Schur complement matrix with 2 subdomains and advection (global Pèclet 5).

For a symmetric operator and a symmetric partition (see figure 4), the symmetric flux splitting is exact and the Neumann-Neumann preconditioner is optimal. The largest discrepancies between the IS preconditioner and the Steklov operator occur at low frequencies and yield a condition number less than two.

If the partition is non-symmetric (see figure 5) then the Neumann-Neumann preconditioner is no longer exact, because $\mathcal{S}^{+} \neq \mathcal{S}^{-}$. However, its condition number is very low whereas the IS preconditioner condition number is still under two. 
For a relatively important advection term, given by a global Pèclet number of 5 (see figure 6), the asymmetry in the flux splitting is much more evident, mainly for small wave numbers, and this results in a large discrepancy between the Neumann-Neumann preconditioner and the Steklov operator. On the other hand, the IS preconditioner is still very close to the Steklov operator.

The difference between the Neumann-Neumann preconditioner and the Steklov operator increases for larger $\mathrm{Pe}$ (see figure 7).

This behavior can be directly verified by computing the condition number of Schur complement matrix and preconditioned Schur complement matrix for the different preconditioners (see tables 1 and 2). We can see that, for low Pe, both the Neumann-Neumann and IS preconditioners give a similar preconditioned condition number regardless of mesh refinement (it almost doesn't change from a mesh of $50 \times 50$ to a mesh of $100 \times 100$ ), whereas the Schur complement matrix exhibits a condition number roughly proportional to $1 / h$. However, the Neumann-Neumann preconditioner exhibits a large condition number for high Pèclet numbers whereas the IS preconditioner seems to perform better for advection dominated problems.

Table 1

\begin{tabular}{|r|r|r|r|}
\hline$P e$ & $\operatorname{cond}(\mathcal{S})$ & $\operatorname{cond}\left(\mathcal{P}_{\mathrm{NN}}^{-1} \mathcal{S}\right)$ & $\operatorname{cond}\left(\mathcal{P}_{\mathrm{IS}}^{-1} \mathcal{S}\right)$ \\
\hline \hline 0 & 41.00 & 1.00 & 4.92 \\
\hline 0.5 & 40.86 & 1.02 & 4.88 \\
\hline 5 & 23.81 & 3.44 & 2.92 \\
\hline 25 & 5.62 & 64.20 & 1.08 \\
\hline
\end{tabular}

Condition number for the Steklov operator and several preconditioners (mesh: $50 \times$ 50 elements, strip: 5 layers of nodes.

\begin{tabular}{|r|r|r|r|}
\hline$u$ & $\operatorname{cond}(\mathcal{S})$ & $\operatorname{cond}\left(\mathcal{P}_{\mathrm{NN}}^{-1} \mathcal{S}\right)$ & $\operatorname{cond}\left(\mathcal{P}_{\mathrm{IS}}^{-1} \mathcal{S}\right)$ \\
\hline \hline 0 & 88.50 & 1.00 & 4.92 \\
\hline 0.5 & 81.80 & 1.02 & 4.88 \\
\hline 5 & 47.63 & 3.44 & 2.92 \\
\hline 25 & 11.23 & 64.20 & 1.08 \\
\hline
\end{tabular}

Table 2

Condition number for the Steklov operator and several preconditioners (mesh: 100× 100 elements, strip: 10 layers of nodes). 


\section{SOLUTION OF THE STRIP PROBLEM}

Efficient implementation of the IS preconditioner in a parallel environment will be the subject of future research. However, we will give some hints here.

A first possibility is a fully coupled, direct solution of the interface problem. This approach involves transferring all the interface matrix to a single processor and solving the problem there. This is not a significant amount of work, but doing it in only one processor would largely unbalance the distribution of load among processors.

A second possibility is partitioning the strip problem among processors, much in the same way as the global problem is. Then, the preconditioning problem can be solved by an iterative method. Care must be taken to avoid nesting a non-stationary method like CG or GMRES inside another outer non-stationary method. The problem here is that a non stationary method executed a finite number of times is not a linear operator, unless the inner iterative method is iterated enough and then approaches the inverse of the preconditioner. In this respect, relaxed Richardson iteration is suitable. The idea of an iterative method is also suggested by the fact that the preconditioning matrix (i.e. the matrix obtained by assembling on the strip domain with Dirichlet boundary conditions at the strip boundary) is highly diagonal dominant for narrow strips. A subsequent possibility is preconditioning the Interface Strip preconditioner problem itself with block Jacobi.

\section{NUMERICAL EXAMPLES}

The performance of the proposed preconditioner is compared in a sequential environment. For this purpose, we consider two different problems. The domain $\Omega$ in both cases is the unit square discretized on an unstructured mesh of $120 \times 120$ nodes, and decomposed in 6 rectangular subdomains. We compare the residual norm versus iteration count by using no preconditioner, NeumannNeumann preconditioner, and the IS preconditioner (with several node layers at each interface side).

The first example is the Poisson's problem $\Delta \phi=g$, where $g=1$ and $\phi=0$ on all the boundary $\Gamma$. The iteration counts and the problem solution (obtained in a coarse mesh for visualization purposes) are plotted in figure 8. As it can be seen, the Neumann-Neumann preconditioner has a very low iteration count, as it is expected for a symmetric operator. The IS preconditioner has a larger iteration count for thin strip widths, but it decreases as the strip is thickened. For a strip of five-layers width, we reach an iteration count comparable to 
the Neumann-Neumann preconditioner with significantly less computational effort. Regarding memory use, the required core memory for thin strip is much less than for the Neumann-Neumann preconditioner. The strip width acts in fact as a parameter that balances the required amount of memory and the preconditioner efficiency.

The second example is an advective-diffusive problem (see 33) at a global Péclet number of $\mathrm{Pe}=u L / 2 \kappa=25, g=\delta(1 / 4,7 / 8)+\delta(3 / 4,1 / 8)$, and $\phi(0, y)=0$. Therefore, the problem is strongly advective. The iteration count and the problem solution (interpolated in a coarse mesh for visualization purposes) are plotted in figure 9. In this example, the advective term introduces a strong asymmetry. The Neumann-Neumann preconditioner is far from being optimal. It is outperformed by IS preconditioner in iteration count (and consequently in computing time) and memory demands, even for thin strips.

\section{CONCLUSIONS}

We have presented a new preconditioner for Schur complement domain decomposition methods. This preconditioner is based on solving a global problem posed in a narrow strip around the inter-subdomain interfaces. Some analytical results have been derived to present its mathematical basis. Numerical experiments have been carried out to show its convergence properties.

The IS preconditioner is easy to construct as it does not require any special calculation (it can be assembled with a subset of subdomain matrices coefficients). It is much less memory-consuming than classical optimal preconditioners such as Neumann-Neumann in primal methods (or Dirichlet in FETI methods). Moreover, it permits to decide how much memory to assign for preconditioning purposes.

In advective-diffusive real-life problems, where the Pèclet number can vary on the domain between low and high values, the proposed preconditioner outperforms classical ones in advection-dominated regions while it is capable to handle reasonably well diffusion-dominated regions.

\section{Acknowledgment}

This work has received financial support from Consejo Nacional de Investigaciones Científicas y Técnicas (CONICET, Argentina, grants PIP 0198/98), Universidad Nacional del Litoral (Argentina, grants CAI+D 2000/43) and ANPCyT (Argentina, grants PICT 6973/99, PID-74/99, PICT Lambda/2003, 
PME 209/2003). We made extensive use of freely distributed software as GNU/Linux OS, MPI, PETSc, gcc compilers, Octave, Open-DX among many others.

\section{References}

[1] V. Sonzogni, A. Yommi, N. Nigro, and M. Storti. A parallel finite element program on a Beowulf cluster. Advances in Engineering Software, 33(7-10):427443, October 2002.

[2] M. Storti, N. Nigro, R. Paz, and L. Dalcín. PETSc-FEM: A general purpose, parallel, multi-physics FEM program. 1999-2006.

[3] R. Paz and M. Storti. An interface strip preconditioner for domain decomposition methods: Application to hydrology. Int. J. Numer. Meth. Eng., 62(13):1873-1894, 2005.

[4] R. Paz, N. Nigro, and M. Storti. On the efficiency and quality of numerical solutions in cfd problems using the interface strip preconditioner for domain decomposition methods. Int. J. Numer. Meth. Fluids., 2005. In press.

[5] Y. Saad. Iterative Methods for Sparse Linear Systems. PWS Publishing Co., 2000 .

[6] P. Le Tallec and M. Vidrascu. Solving large scale structural problems on parallel computers using domain decomposition techniques. In M. Papadrakakis, editor, Parallel Solution Methods in Computational Mechanics, chapter 2, pages 49-85. John Wiley \& Sons Ltd., 1997.

[7] FX. Roux, F. Magoulès, L. Series, and Y. Boubendir. Approximations of optimal interface boundary conditions for two-lagrange multiplier FETI method. In 15th International Conference on Domain Decomposition Methods, 2003.

[8] J. Mandel. Balancing domain decomposition. Comm. Appl. Numer. Methods, 9:233-241, 1993.

[9] J.M. Cros. A preconditioner for the Schur complement domain decomposition method. In 14 th International Conference on Domain Decomposition Methods, 2002 .

[10] F. Bourquin and N. Rabah. Decoupling and modal synthesis of vibrating continuos systems. In 9th International Conference on Domain Decomposition Methods, 1996.

[11] C. Farhat and F.X. Roux. A method of finite element tearing and interconnecting and its parallel solution algorithm. Int. J. Numer. Meth. Eng., 32:1205-1227, 1991. 
[12] C. Farhat, J. Mandel, and F.X. Roux. Optimal convergence properties of the FETI domain decomposition method. Comput. Meth. Appl. Mech. Engrg., 115:365-385, 1994.

[13] C. Farhat and J. Mandel. The two-level FETI method for static and dynamic plate problems. Comput. Meth. Appl. Mech. Engrg., 155:129-152, 1998.

[14] C. Farhat, M. Lesoinne, P. Le Tallec, K. Pierson, and D. Rixen. FETI-DP: a dual-primal unified FETI method-part I: A faster alternative to the two-level FETI method. Int. J. Numer. Meth. Eng., 50:1523-1544, 2001. 


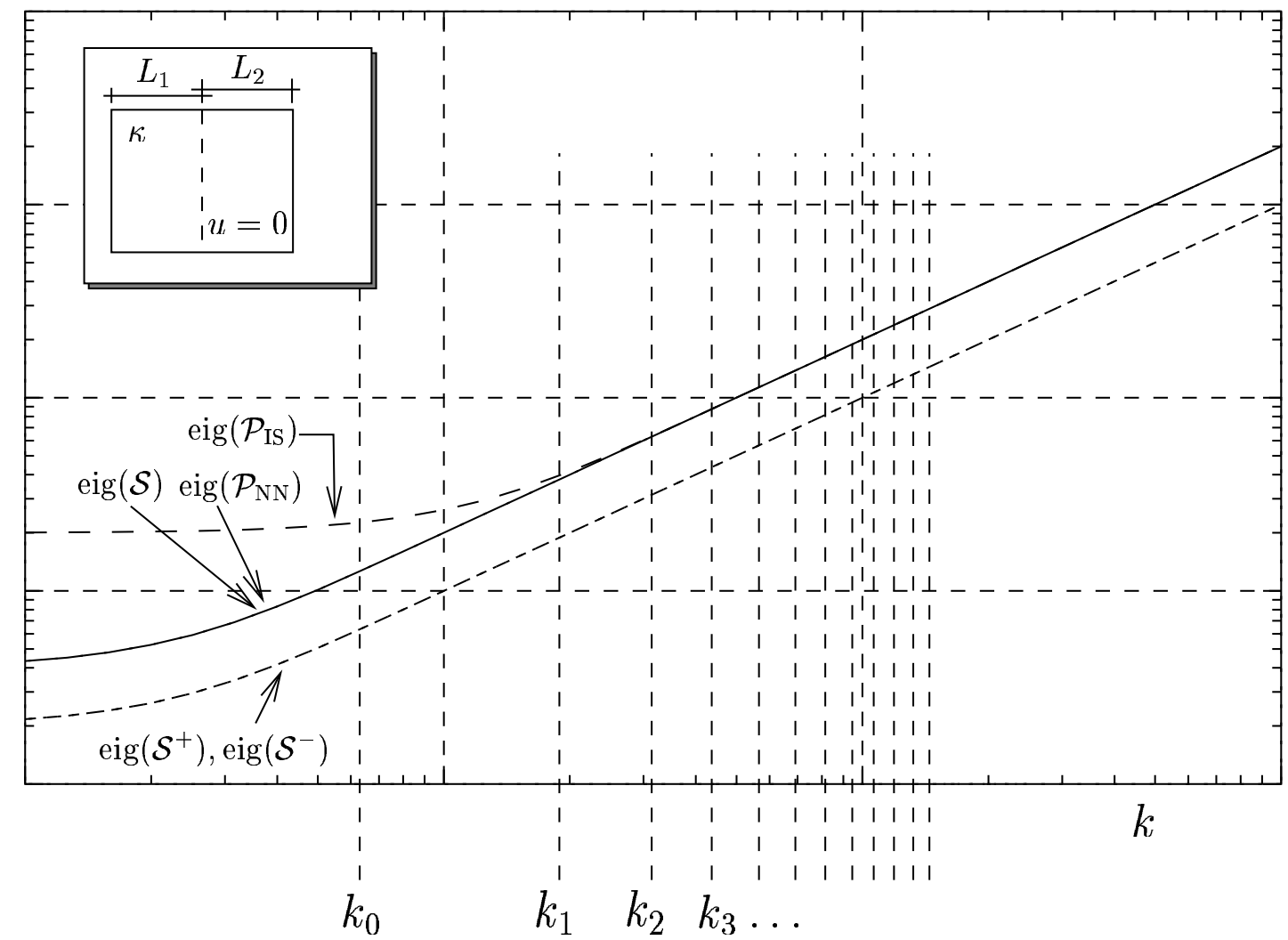

Fig. 4. Eigenvalues of Steklov operators and preconditioners for the Laplace operator $(P e=0)$ and symmetric partitions $\left(L_{1}=L_{2}=L / 2, b=0.1 L\right)$. 


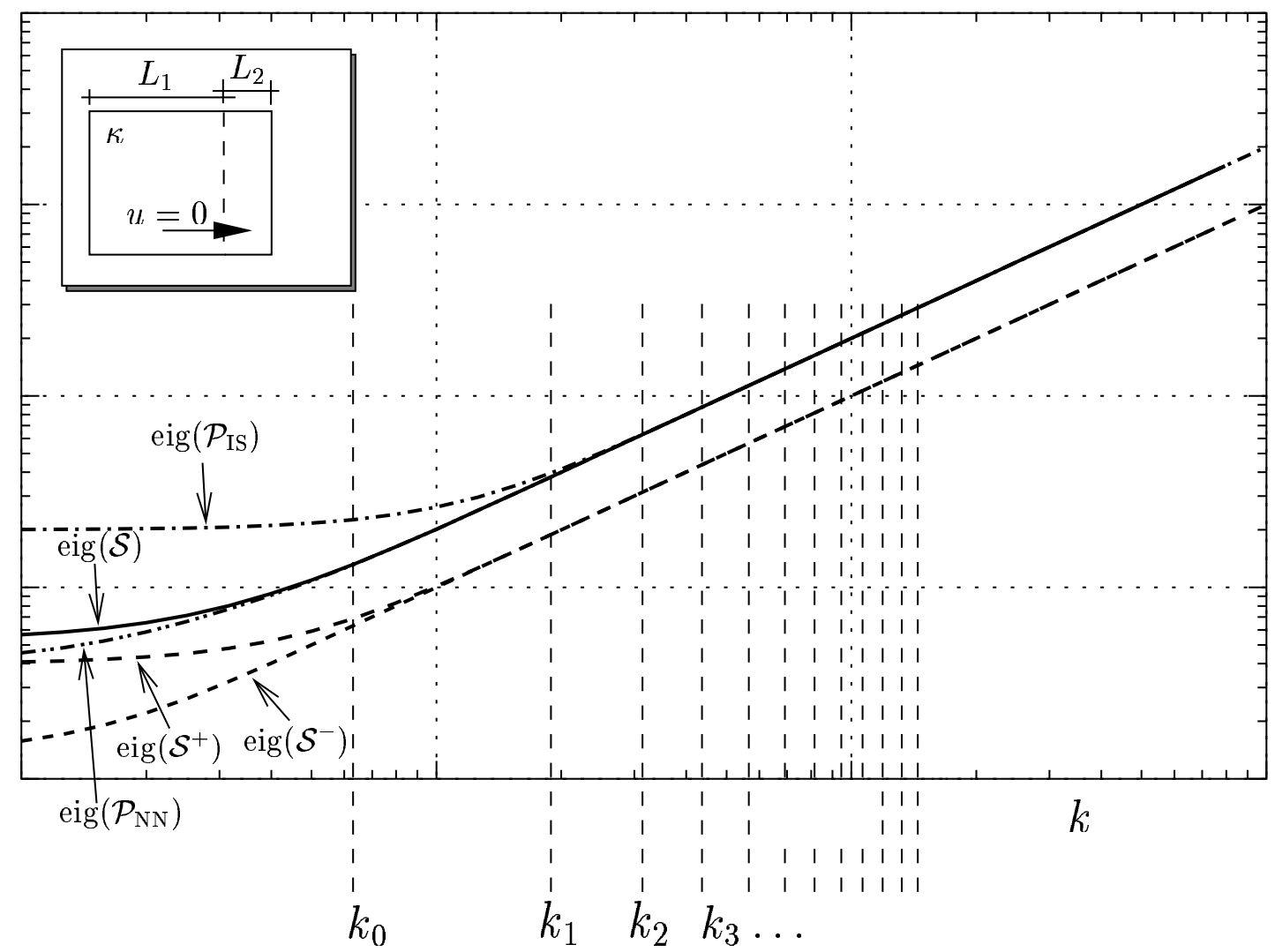

Fig. 5. Eigenvalues of Steklov operators and preconditioners for the Laplace operator $(P e=0)$ and non-symmetric partitions $\left(L_{1}=0.75 L, L_{2}=0.25 L, b=0.1 L\right)$. 


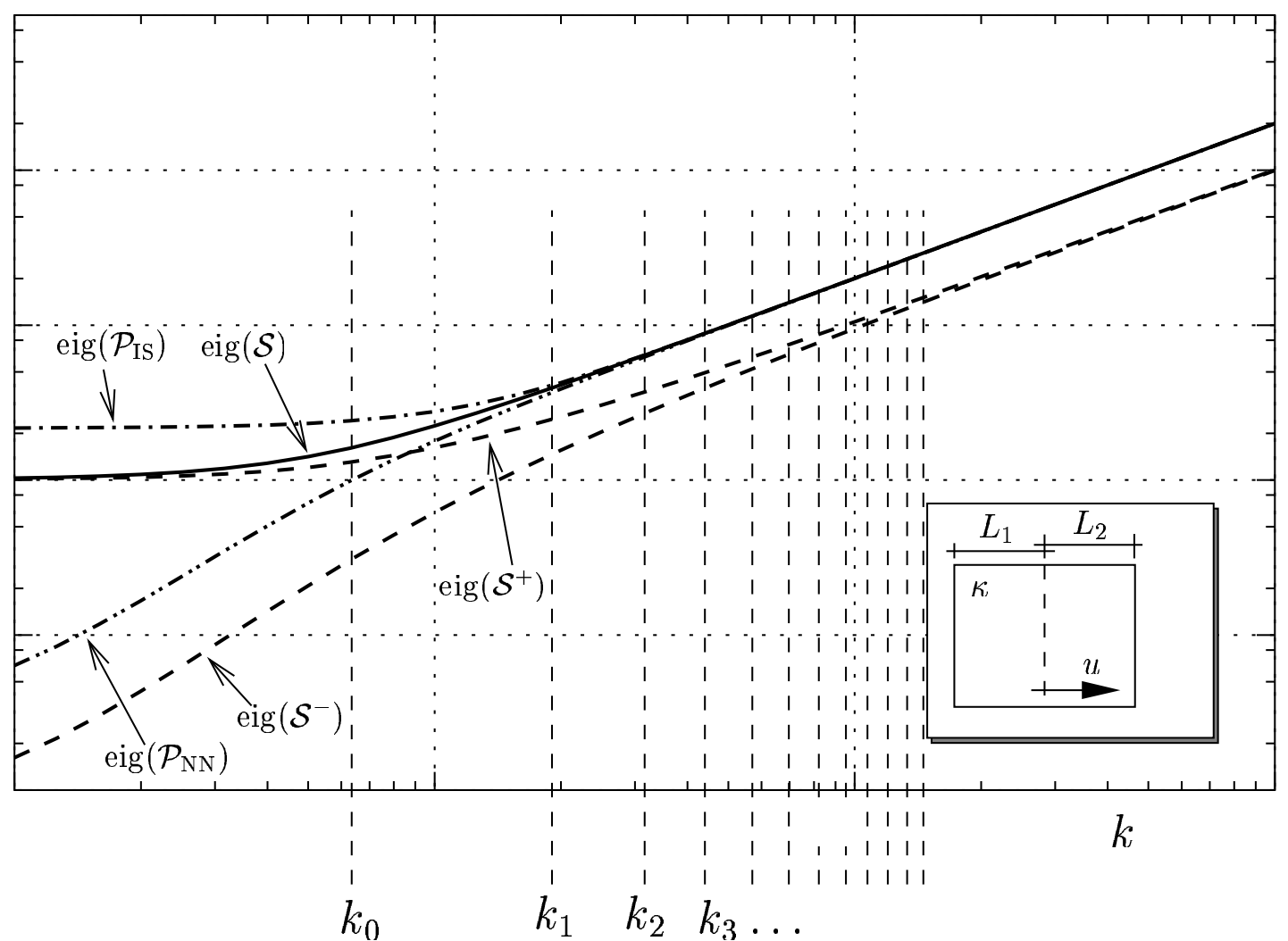

Fig. 6. Eigenvalues of Steklov operators and preconditioners for the advection-diffusion operator $(\mathrm{Pe}=5)$ and symmetric partitions $\left(L_{1}=L_{2}=L / 2, b=0.1 L\right)$. 


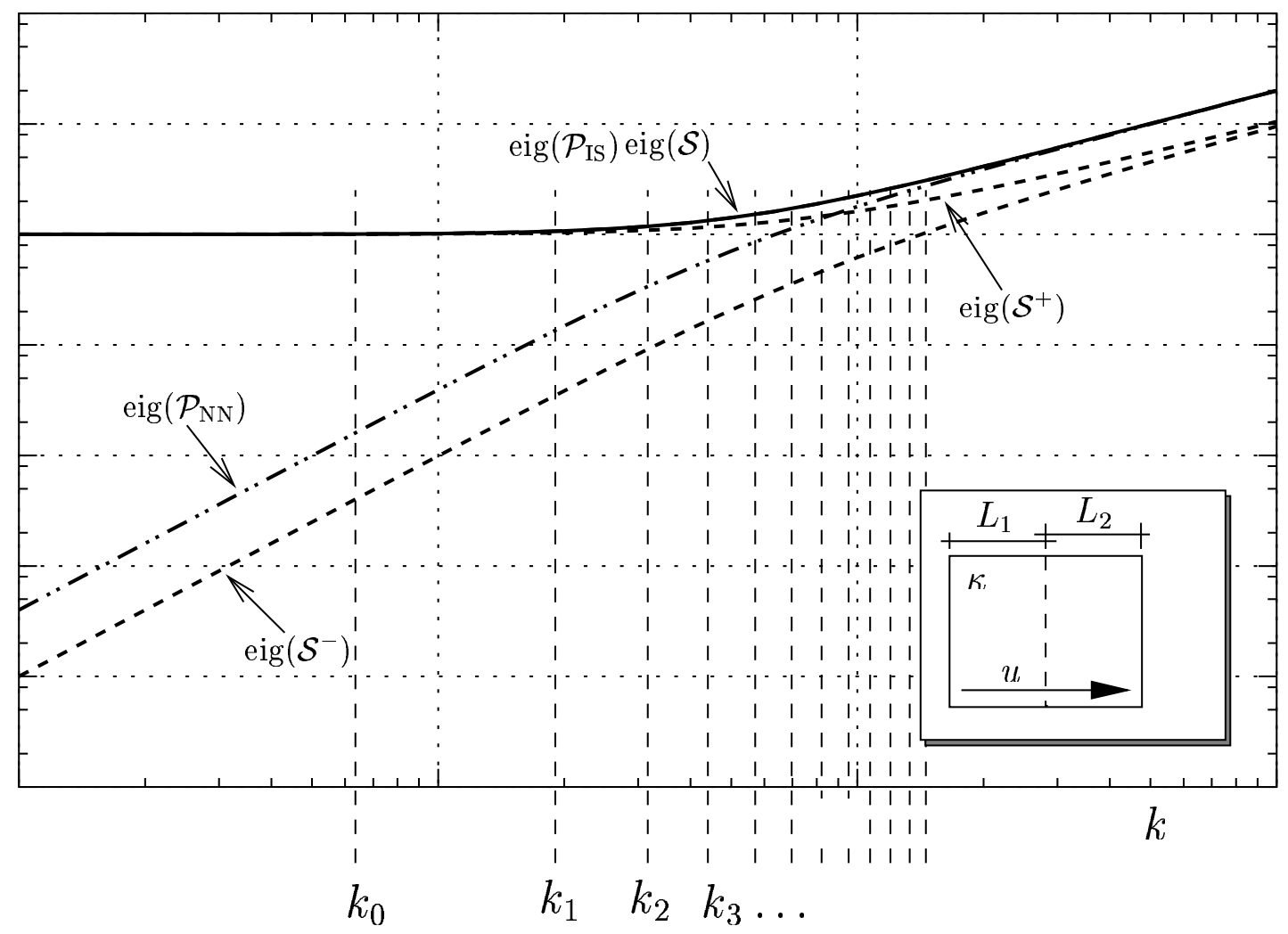

Fig. 7. Eigenvalues of Steklov operators and preconditioners for the advection-diffusion operator $(\mathrm{Pe}=50)$ and symmetric partitions $\left(L_{1}=L_{2}=L / 2, b=0.1 L\right)$. 


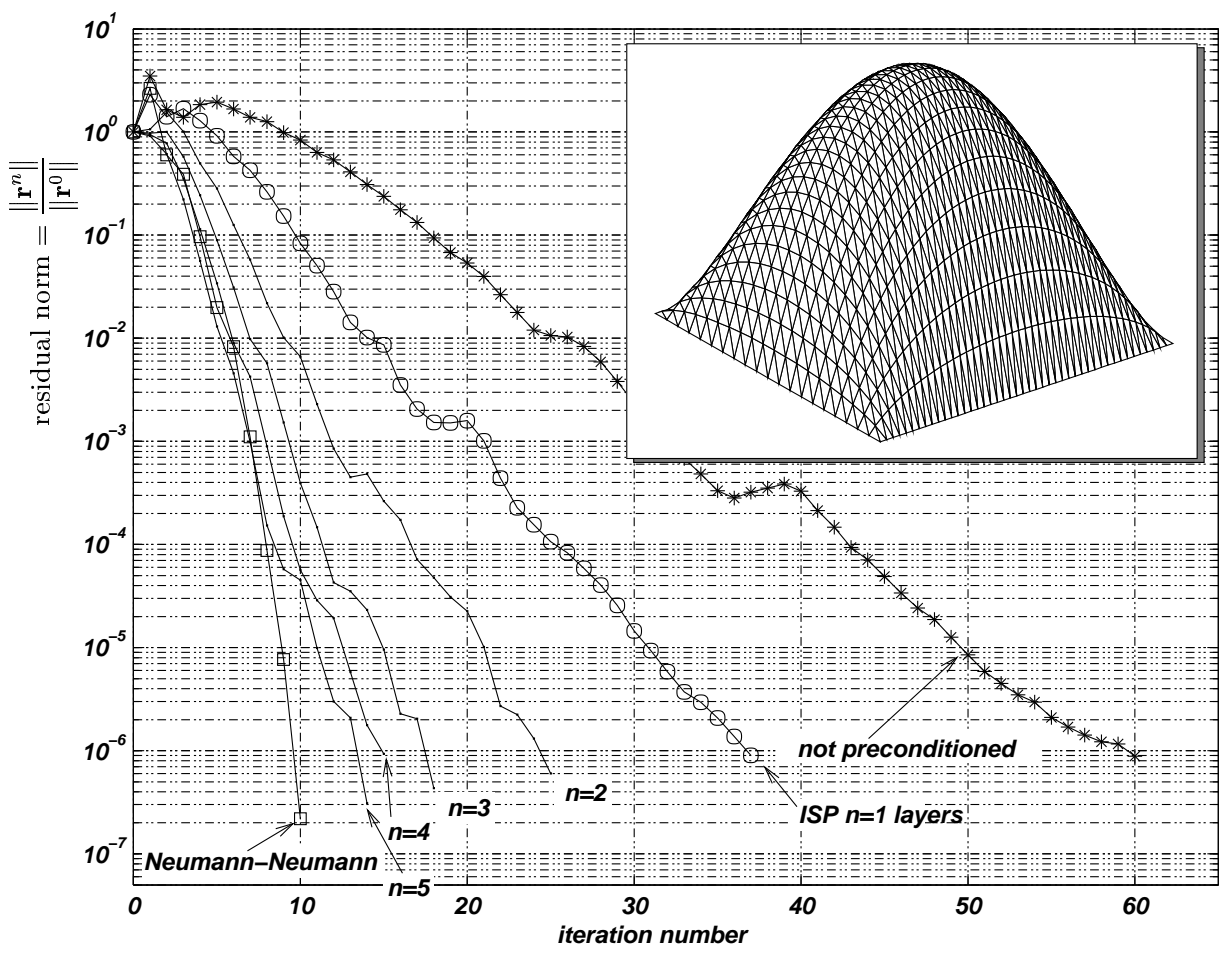

Fig. 8. Solution of Poisson's problem.

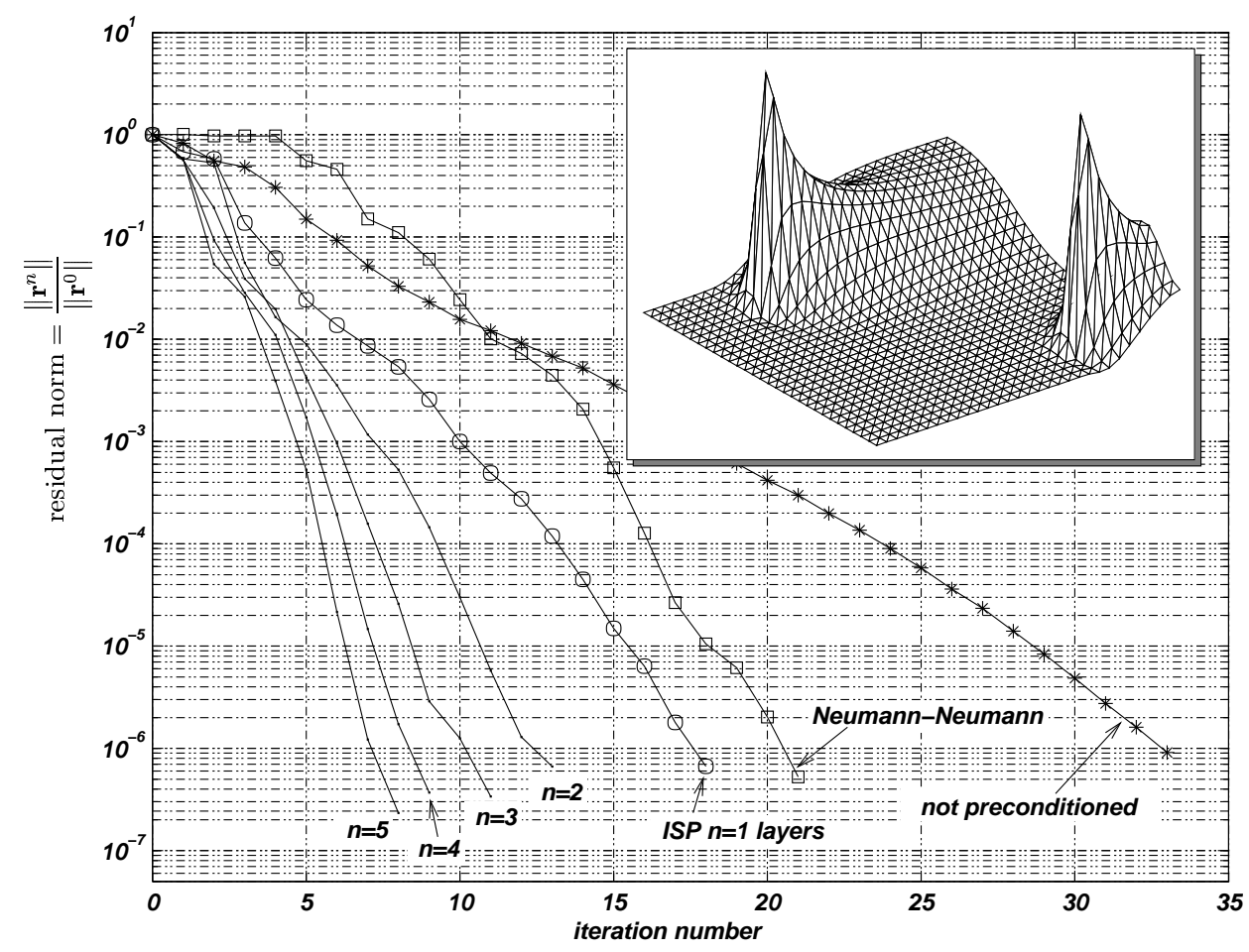

Fig. 9. Solution of advective-diffusive problem. 